\title{
Iris Recognition in Mobile Phone Based on Adaptive Gabor Filter
}

\author{
Dae Sik Jeong ${ }^{1}$, Hyun-Ae Park ${ }^{1}$, Kang Ryoung Park ${ }^{2}$, and Jaihie Kim ${ }^{3}$ \\ ${ }^{1}$ Department of Computer Science, Sangmyung University, \\ 7 Hongji-Dong, Jongro-ku, Seoul, Republic of Korea \\ Biometrics Engineering Research Center (BERC) \\ \{jungsoft97, whitebbb\} asmu.ac.kr \\ ${ }^{2}$ Division of Media Technology, Sangmyung University, \\ 7 Hongji-Dong, Jongro-ku, Seoul, Republic of Korea \\ Biometrics Engineering Research Center (BERC) \\ parkgr@smu.ac.kr \\ ${ }^{3}$ Biometrics Engineering Research Center (BERC), \\ Department of Electrical and Electronic Engineering, Yonsei University, \\ Seoul, Republic of Korea \\ jhkimeyonsei.ac.kr
}

\begin{abstract}
As the security of personal information is becoming more important in mobile phones, we apply iris recognition technology to mobile device. Different from conventional iris recognition system used for access control, user puts the mobile phone by hands in this case. So, optical and motion blurring happens, frequently. In addition, most users have tendencies to use the mobile phone in outdoor and sunlight (which includes much amount of IR(Infra-Red) light) may have much effect on the input iris image in spite of the visible light cut filter attached in front of iris camera lens. To overcome such problems, we propose a new method of extracting the accurate iris code based on AGF (Adaptive Gabor Filter). The kernel size, frequency and amplitude of Gabor filter are determined by the amount of blurring and sunlight in input image, adaptively. Experimental results show that the EER by our propose method is $0.14 \%$.
\end{abstract}

\section{Introduction}

Compared to other biometric systems, the iris recognition system is reputed to the most reliable among all biometric methods [3][4][5][6][7]. Iris recognition is to recognize a person by using unique iris patterns, which exist in iris region between white sclera and black pupil [1][2].

Recently, with the increasing need of guaranteeing the security in case of using bank transaction service by using mobile phone, it is required to apply biometrics for the security of mobile phone. For example, mobile phone with fingerprint recognition (such as LG-KP3800 made by LG Electronic) has been already produced [9]. However, it requires additional fingerprint image acquisition sensor and DSP chip for fingerprint recognition. This cause the cost and size of mobile phone to be increased. So, we try to apply the iris recognition to mobile phone. 
In general, for iris recognition, the magnified iris image (more than 200 pixels of diameter) is required for accurate recognition [13]. So we need a zoom and focus lenses for iris recognition camera, which cause the size and cost to be increased. However, in case of using mega pixel camera built in mobile phone, though face image is captured with the far distance between eye and camera (more than 30 to 40 $\mathrm{cm}$ ), the iris region can include sufficient pixel information for recognition. So, we aim at developing the iris recognition system in mobile phone only by using a built-in mega-pixel camera and software without additional hardware component.

For iris recognition, iris feature extraction is critical step. Previous methods for iris feature extraction [3][4][7][8][14] require too much processing power to be used in ARM CPU in mobile phone (for example, ARM 9 has the processing power of about $200 \mathrm{MHz}$ [16] [17] and ARM CPU does not have an internal floating point processing). Otherwise, Noh's method [15] uses ICA based 1D Filter. However, it uses the determined ICA basis from training and cannot deal with the iris image affected by image blurring and sunlight in mobile phone. To overcome such problems, we proposed a new 1D Adaptive Gabor Filter apt for iris recognition in mobile phone.

\section{Iris and Pupil Localization}

To extract iris code from input iris image, we first detect iris and pupil region. Because the pupil has the hollow structure inside of cornea [18], the gray level of pupil in input image is very low compared to other region such as iris, sclera and skin. So, by simple binarization, we can discriminate the pupil and the other region such as iris, sclera and facial skin. However, some dark region such as shaded facial skin, iris and eyelash shows same gray level to pupil. So, we change decoder value of brightness and contrast, if we make the brightness and contrast value of $\mathrm{A} / \mathrm{D}$ converter lower and higher, we can easily select the binarization threshold to detect the pupil region and bright corneal specular reflection [19]. From that information, we detect the pupil region and locate iris region by modified circular edge detection [19]. We then choose a region free from occlusions caused by eyelids or eyelashes. To detect eyelashes, we use the eyelash mask [25]. We also use the elliptical eyelid deformable template to detect the eyelid regions [26]. The iris codes generated in eyelash and eyelid are set to invalid codes and are not used for enrollment and recognition.

\section{Adaptive Gabor Filter Considering Blurring and Sunlight}

\subsection{Overview of the Proposed Adaptive Gabor Filter and the Illuminator- On/Off Scheme}

After detecting iris region in input image, we extract iris feature code by Adaptive Gabor Filter. The overview of the proposed method is shown in Fig. 1. As mentioned before, most users of mobile phone have tendencies to use the mobile phone in outdoor and sunlight (which includes much amount of IR(Infra-Red) light) may have much effect on the input iris image in spite of the visible light cut filter attached in front of iris camera lens. In addition, different from conventional iris recognition system used for access control, user puts mobile phone by hand and optical \& motion 
blurring happen frequently. So, we measure the amount of blurring and sunlight from input image and change the kernel size, frequency and amplitude of Gabor filter according to the measure amount.

As shown in Fig. 1, user presses "the button for iris recognition" in the mobile phone and the camera micro-controller turns the IR (Infra-Red) illuminator on and off, successively, and six successive iris images are captured as shown in Fig. 1(1) and 2.

Then, we measure the amount of sunlight in input image ((2) in Fig. 1). For that, we calculate a average gray value of image frame \#2, \#4 and \#6 (of Fig.2) as Eq.(1). As shown Fig. 2, because image frame \#2, \#4 and \#6 are obtained with IR illuminator off and we use visible light cut filter in front of camera lens, the image is bright in case that environmental sunlight exist.

$$
B r=(\mathrm{A} 2+\mathrm{B} 2+\mathrm{C} 2) / 3 \quad(0 \leq B r \leq 255),
$$

where A2, B2 and C2 are a gray value in image frame \#2, \#4 and \#6, respectively. In case that $\boldsymbol{B r}$ exceeds in 50, we determine the outdoor environment in which sunlight exists. Otherwise, we do the indoor environment in which sunlight does not exist.

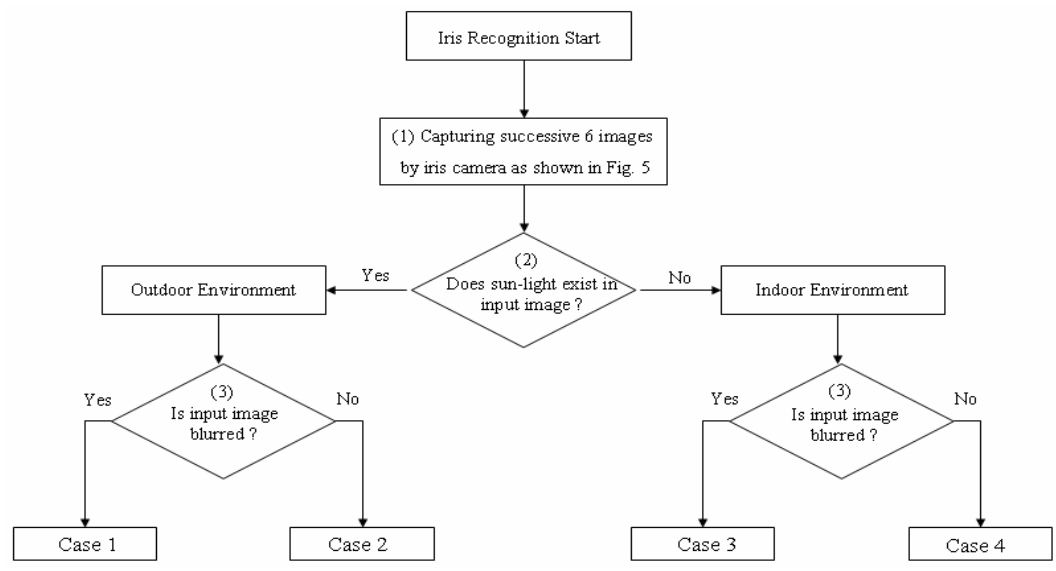

Fig. 1. Overview of proposed method

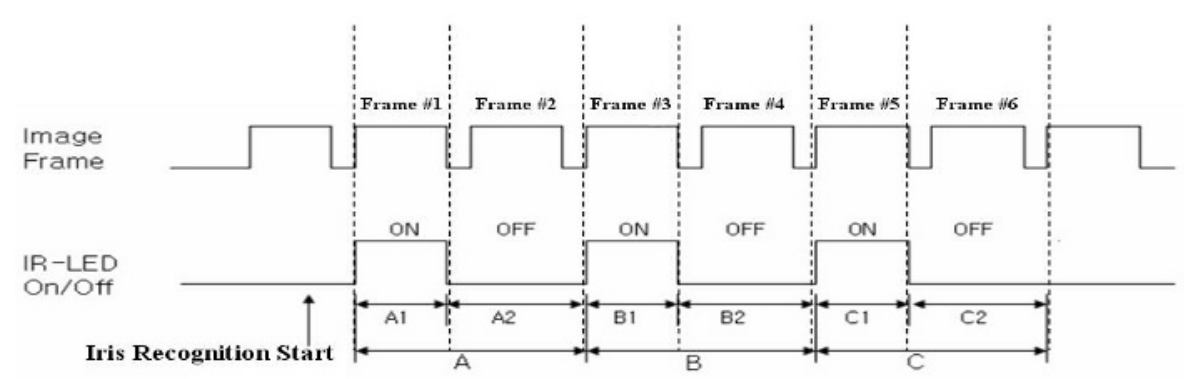

Fig. 2. Capturing successive 6 images by iris camera in mobile phone 
Then we check whether the input image is blurred or not ((3) in Fig. 1). For that, we use the iris focus checking mask proposed by Kang [25], which checks high frequency component in input image. With that, we can obtain continuous focus value from 0 to 100 (100 means the most focused and 0 does the least focused). We apply the focus checking mask to the image frame \#1 and \#3 (of Fig.2), and obtain the focus value of FV1 and FV2 in Eq. (2). And final focus value in input image is calculated by $\boldsymbol{F t}$ and in case that $\boldsymbol{F t}$ exceeds in 50, we determine there exist optical \& motion blurring in input image.

$$
F t=(\text { FV1 }+ \text { FV2 }) / 2 \quad(0 \leq F t \leq 100),
$$

where FV1 and FV2 are the measured focus value of A1 and B1. As shown in Fig. 1, we can classify 4 cases as such (Case 1 : Blurred and Sunlight, Case 2 : Focused and Sunlight, Case 3 : Blurred and No Sunlight, Case 4 : Focused and No Sunlight)

\subsection{Adaptive Gabor Filter}

Then, we use the measured $\boldsymbol{B r}$ and $\boldsymbol{F t}$ for selecting the kernel size, frequency and amplitude of Gabor filter. In our case, we use adaptive Gabor Filter that can be defined as follows:

$$
G(x)=A \cdot e^{-\pi\left[\frac{\left(x-x_{0}\right)^{2}}{\sigma^{2}}\right]}\left(\operatorname { c o s } \left(2 \pi\left[u_{0}\left(x-x_{0}\right]\right), \sum_{x_{0}=-N}^{N} G(x)=0(\because D C=0),\right.\right.
$$

(To make iris code generation is not affected by the image brightness of iris texture, we set the DC component of Gabor filter to be 0)

where $\boldsymbol{A}$ is Gabor Filter's amplitude, $\sigma$ and $\boldsymbol{u}_{\boldsymbol{0}}$ are the kernel size and the frequency of Gabor Filter, respectively. $2 N$ is the number of Gabor Filter coefficient. Due to the limitation of processing power in mobile phone, we only use the real term of Gabor Filter. In addition, we do not use the scheme of iris region stretching to make rectangle iris region. Instead, we extract the iris code directly from iris region of polar coordinate. To consist with conventional Daugman iris code structure, we use 8 tracks and 256 sectors to extract iris code. After extracting iris code, we use the HD (Hamming Distance) to measure the dissimilarity between two iris codes [3].

The gray level change (contrast) in iris texture is diminished in case of sunlight. That is because sunlight increases the overall image brightness of iris texture. In addition, when the longer wavelength (more than 900nm) of IR illuminator is used by sunlight (the wavelength of our iris camera illuminator is 760 and $850 \mathrm{~nm}$, but sunlight includes much amount of IR component of longer wavelength), iris texture shows the characteristics of low contrast. In such case, the accurate iris code cannot be extracted by static Gabor filter. Especially, in case that user enrolls his iris in indoor and tries to identify himself in outdoor, the FRR may be increased. To overcome such problem, we increase the amplitude of Gabor filter in case of sunlight, which has the effect that increases the signal level of low contrast iris texture.

To determine the amplitude $(\boldsymbol{A})$ of Gabor filter, we use the measured the image brightness by sunlight $(\boldsymbol{B r})$ as shown in Eq. (4)

$$
\boldsymbol{A}=(1 / 11) \boldsymbol{B} \boldsymbol{r}+180
$$


Eq.(4) was obtained by experiment. About each image brightness (Br), we measured the EER with Gabor filter (Eq.(3)) of various range of amplitude and selected the optimal amplitude $(\boldsymbol{A})$ in each image brightness $(\boldsymbol{B r})$. In this case, we used a static kernel size of 47 and frequency of $\pi / 8$, which were obtained by our experiment with CASIA DB [23] of showing the minimum EER. Of course, the phase component of Gabor filter is not basically affected by the iris image contrast, but we use the offset margin for quantizing of iris code ( 0 or 1$)$ instead of using threshold 0 . So, in case that image contrast is low due to sunlight, the calculated phase component is very small and cannot be credible with offset margin. Due to such reason, Eq.(4) can be useful in case of iris texture of low image contrast. In similar method, we obtain the kernel $\operatorname{size}(\boldsymbol{\sigma})$ and frequency of Gabor filter $\left(\boldsymbol{u}_{\boldsymbol{0}}\right)$ based on the focus value $\boldsymbol{F t}$ of Eq. (2) as shown in Eq.(5). In general, the more the input image is blurred, the longer the kernel size and lower frequency of Gabor filter is required to deal with low frequency iris texture.

$$
\begin{aligned}
\sigma & =(-1 / 16) \boldsymbol{F} t+54 \\
\boldsymbol{u}_{\boldsymbol{0}} & =(-\pi / 800) \boldsymbol{F} \boldsymbol{t}+\pi / 4
\end{aligned}
$$

From that, we can determine the amplitude and the kernel size \& frequency of Adaptive Gabor Filter based on the measured image brightness and focus value and extract the accurate iris code for recognition.

\section{Experimental Results}

For experiment, we use the mobile phone (the model name is SPH-S2300 (by Samsung Electronics) with a 3.2 mega-pixel (2048*1536) CCD sensor). To capture detailed iris patterns, it is necessary to use an IR-illuminator and an IR pass filter [16][17]. We attached the IR pass filter (which only passes over the wavelength of $750 \mathrm{~nm}$ ) in front of the camera lens and used the built-in Xenon flash lamp (which had the characteristics of including both visible and IR light wavelengths) for iris recognition. Tests were performed on 200 iris images captured from 20 persons. Test images were also obtained indoors (100 images) and outdoors (100 images). The average intensity of illumination in the indoor was 223 Lux. (measured by optical power meter) and that in the outdoor was 1,394 Lux. (including sunlight). Experimental results showed that in case of using Daugman's Gabor filter (with the kernel size of 47 , the frequency of $\pi / 8$ and amplitude of $-120 \sim+170)$, the EER was $0.09 \%$ ( $\boldsymbol{d}$ ' is 8.4 [8]) (all the enrollment and recognition were performed with the indoor images) and $0.11 \%$ ( $\boldsymbol{d}^{\prime}$ is 8.1 ) (all the enrollment and recognition were performed with the outdoor images). However, the EER was much increased to $1.2 \%$ ( $\boldsymbol{d}^{\prime}$ is 1.5 ) (the enrollment was performed with indoor images, but the recognition was tried with outdoor images of sunlight). In this case, optimal kernel size, frequency and amplitude of Gabor filter were obtained using CASIA DB [23]. The CASIA DB consists of 8-bit gray-level images of 756 (108 eyes for 80 subjects) with 320x280 pixels. The EER was $0.088 \%$ ( $\boldsymbol{d}^{\prime}$ is 8.5 [8]) (all the enrollment and recognition were performed with the CASIA DB) and we can know that iris image quality of our mobile camera is not degraded compared to CASIA DB excluding sunlight and severe blurring. In case 
of using Adaptive Gabor Filter based on the measured image brightness and focus value, the EER was $0.09 \%$ ( $\boldsymbol{d}^{\prime}$ ' is 8.4 ) (all the enrollment and recognition were performed with the indoor images) and $0.10 \%$ ( $\boldsymbol{d}^{\prime}$ 'is 8.2 ) (all the enrollment and recognition were performed with the outdoor images). However, the EER was somewhat increased to $0.14 \%$ ( $d^{\prime}$ is 7.7 ) (the enrollment was performed with indoor images, but the recognition was tried with outdoor images of sunlight). However, it is much smaller to that with static Gabor filter. In this case, we set the threshold for authentic and imposter distribution, with which the FAR is equal to the FRR, by experiment. Processing time is about $1,800 \mathrm{~ms}$ in Mobile Phone (SPH-S2300) of ARM926EJ-STM (150 MHz).

\section{Conclusions}

In this paper, we propose a new method of extracting the accurate iris code based on AGF (Adaptive Gabor Filter). The kernel size, frequency and amplitude of Gabor filter are determined by the amount of blurring and sunlight in input image, adaptively. Experimental results show that the EER by propose method is $0.14 \%$. In future works, more field tests are required to enhance the performance of AGF. In addition, the eyelash shade region made by sunlight should be detected and excluded to extract iris code for better performance.

\section{Acknowledgements}

This work was supported by the Korea Science and Engineering Foundation (KOSEF) through the Biometrics Engineering Research Center (BERC) at Yonsei University.

\section{References}

[1] A. K. Jain, et al., "BIOMETRICS: Personal Identification in Networked Society," Kluwer Academics Publishers, Norwel, MA, 1999

[2] A. Pankanti, R. M. Bolle and A. K. Jain, "Biometrics: the future of identification," IEEE Computer, Vol. 33, No. 2, pp. 46-49, 2000.

[3] J. G. Daugman, "High Confidence Visual Recognition of Personals by a Test of Statistical Independence ", IEEE Trans. PAMI, Vol.15, No.11, pp.1148 - 1160, Nov., 1993.

[4] Richard P. Wildes, "Iris Recognition:An Emerging Biometrics Technology", Proceedings of the IEEE, Vol.85, No.9, pp.1348 - 1363, Sep., 1997.

[5] W.W. Boles and B. Boashash, "A Human Identification Technique Using Image of the Iris and Wavelet Transform”, IEEE Trans. on SP, Vol.56, No.4, pp.1185-1188, 1998.

[6] Christel-loic, et al., "Person identification technique using human iris recognition", The 15th International Conference on Vision Interface, May, pp.294-299, 2002.

[7] Yong Zhu, et al., "Biometric personal identification based on iris patterns", Proceedings of 15th International Conference on Pattern Recognition, Sep., 2000, Vol. 2, pp. 805-808

[8] John G. Daugman, "The importance of being random: statistical principle of iris recognition", Pattren Recognition, vol. 36, no. 2, pp279-291

[9] http://www.lge.com (accessed on June 8, 2005) 
[10] http://www.lgiris.com (accessed on June 8, 2005)

[11] http://www.iridiantech.com (accessed on June 8, 2005)

[12] http://www.panasonic.com/cctv/products/biometrics.asp (accessed on June 8, 2005)

[13] Kang Ryoung Park, Jaihie Kim, "A Real-time Focusing Algorithm for Iris Recognition Camera", Lecture Notes in Computer Science (ICBA 2004), Vol. 3072, pp.410 417, July 2004

[14] Li Ma, et al., "Personal Identification Based on Iris Texture Analysis", IEEE Trans. PAMI, Vol. 25, No. 12, Dec. 2003

[15] S.I.Noh, et al.,"A New Iris Recognition Method Using Independent Component Analysis", IEICE Transactions on Information and Systems, accepted for publication

[16] http;//www.arm.com (accessed on June 8, 2005)

[17] http://www.qualcom.com (accessed on June 8, 2005)

[18] Gregory A. Baxes, Digital Image Processing - Principles and Applications, Wiley

[19] Dal Ho Cho, et al., "Real-time Iris Localization for Iris Recognition in Cellular Phone", SNPD2005, Towson University, Maryland, USA, May 23 - 25, 2005

[20] http://www.biometrics.org (accessed on June 8, 2005)

[21] http://ww.iris-recognition.org (accessed on June 8, 2005)

[22] John. Dagman, "How Iris Recognition Work", IEEE Trans. on Circuits and Systems Video Technology, Vol. 14, No. 1, Jan. 2004

[23] CASIA iris DB of Chinese Academy of Science, http://www.sinobiometrics.com/resources.htm (accessed on June 8, 20)

[24] R. Jain, Machine Vision, McGraw-Hill

[25] Byung Joon Kang, Kang Ryoung Park, “A Study on Iris Image Restoration”, Lecture Notes in Computer Science (AVBPA 2005), July 2005, accepted for publication

[26] Kang Ryoung Park, "Practical Gaze Point Computing Method by 3D Position Estimation of Facial and Eye Features", LNCS, Vol. 3339, pp.237 247, Dec., 2004 